\title{
Zigbee Based Student Attendance System Using Lan Networking
}

\author{
Prajakta S. Joshi ${ }^{1}$, Shubham S.Joshi ${ }^{2}$ \\ ${ }^{1}$ Aditya Engineering Collage, Assistant Professor, Department Of Electronics And Telecommunication, \\ Beed, Maharashtra \\ ${ }^{2}$ Maharashtra Institute of Technology, Department Of Electronics And Telecommunication,
} Pune, Maharashtra.

\begin{abstract}
In this paper provides the design method of wireless fingerprint attendance system based on ZigBee technology using LAN networking. The system includes terminal fingerprint acquisition module and attendance management module through computer. It can realize automatically such functions as information acquisition of fingerprint, processing, wireless transmission, fingerprint matching, and attendance management. Considering the fact and topology of ZigBee network, a fingerprint acquisition module and a wireless alarm module were designed by using the fingerprint sensor and module respectively. The whole system was implemented wireless alarm through messages and internet in the GSM web. All HODs PCs are worked as workstation and LAN connected with the Principal office. In order to achieve the simple and high real-time system, it realized lowcost and high-performance wireless fingerprint attendance function, which provided a new wireless fingerprint attendance system for enterprises and institutions.
\end{abstract}

Keywords: Zibee Technology, Fingerprint Identification, Wireless Transmission, Wireless Alarm, LAN Networking

\section{Introduction}

The most common means of tracking student attendance in the classroom is by enforcing the students to manually sign the attendance sheet,

which is normally passed around the classroom while the lecturer is conducting the lecture. There are numerous disadvantages of using such system. The attendance sheet is passed around the class; some students may accidentally or purposely sign another student's name. Another issue of having the attendance record in a hardcopy form is that a lecturer may lose the attendance sheet. As a consequence of that, lecturer can no longer trace the students overall attendance record throughout the particular semester.

In this system students report their attendance via biometric system and notification of attendance is passed to HOD office through Zigbee Module. All HOD PC's are LAN connected to the Principal office. So that Principal get a master copy of whole day activity and can know about the every lecture conducted by concern faculty and take action on lecturer who does not taken lecture. The fingerprint has a lot of advantages, such as unique, permanent, good anti-fake and easy to use. So it is recognized increasingly by people.

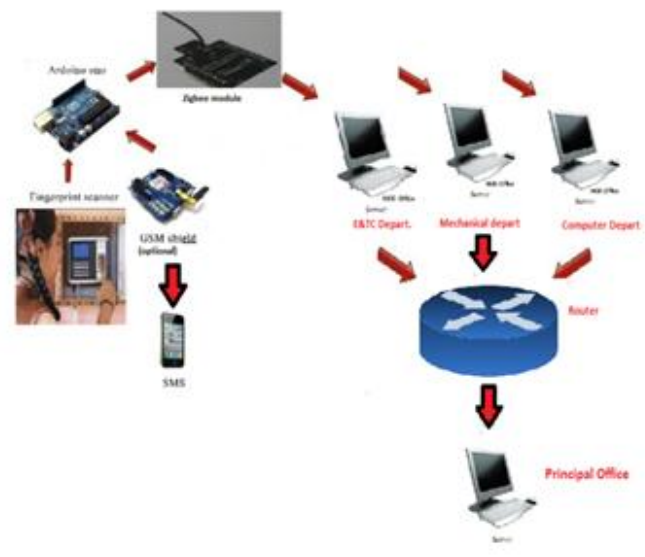

Fig. 1: Overall System Architecture

In above architecture we can see the student place his/her attendance on Fingerprint Scanner and then the chip matches present entry and database which is stored in memory if it gets ok then it will proceed to wireless Zigbee module. Two Zigbee modules are there one at transmitter and another at receiver i.e. HOD Office. In above architecture we have shown Three departments like Electronics, Mechanical and Computer. After collecting data on individual department HOD cabins, then that data will be pass to principal cabin which 
is connected using Local Area Networking through router. So that Principal can get the knowledge about the lectures conducted in a day. So the master report will be generated to the principal office. And then principal can take action against the lecturers who does not conduct the lecture. And keeps the permanent record of College of whole semester. There is one optional module is present that is GSM module. If HOD wants to inform detained students parents then that module can be activated to send SMS of weekly or monthly report to the students and their parents mobile.

\section{Comparison With Other Student Attendance Systems}

There are various other kinds of student attendance management systems available like RFID based student attendance system and GSM-GPRS based student attendance system. These systems have their own pros and cons. Our system is better because first it saves time that could be used for teaching. Second is portability has its own advantage because the device could be taken to any class wherever it is scheduled. While GSMGPRS based systems use position of class for attendance marking which is not dynamic and if schedule or location of the class changes, wrong attendance might be marked. Problem with RFID based systems is that students have to carry RFID cards and also the RFID detectors are needed to be installed. Nonetheless, students may give proxies easily using friend's RFID card. These problems are not in our system. We used fingerprints as recognition criteria so proxies Cannot be given. If portable devices are used, attendance marking will be done at any place and any time. So our student attendance system is far better to be implemented.[1]

III. System Development

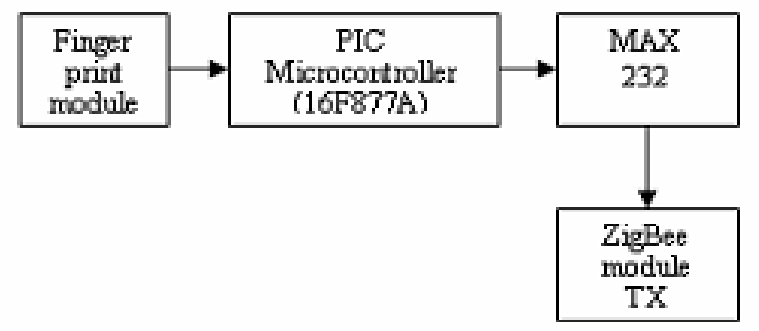

Fig. 2a). Transmitter block diagram

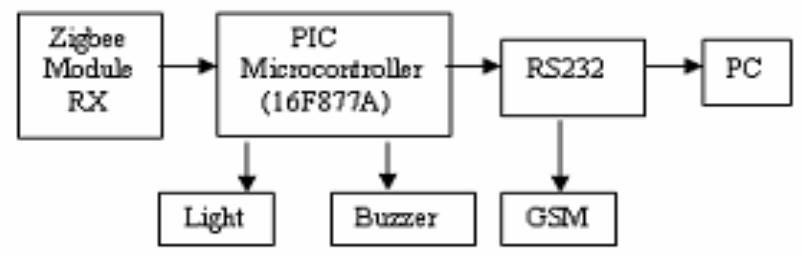

Fig. 2 b). Receiver block diagram

\section{Working}

The system consists of fingerprint acquisition module, Transmission and receiving module, and attendance Management workstation. Fingerprint acquisition module is used to realize fingerprint collecting and pre-treatment Transmission and receiving ZigBee module is used to send the finger print image to computer. Attendance management workstation is used to realize fingerprint extraction and matching in order to realize attendance function. If the finger prints feature and some sample in the database matches, access is permitted, or there will be alarm. There into, alarms are divided into local alarms and wireless ones. Local alarm is given by sound and light lamps. Wireless alarm send messages to the concerned mobile phone by GSM module through which connect GSM network. And it is also sent to The PC alarm monitor.

\subsection{Zibee Module}

ZigBee is a low-cost, low-power, wireless mesh network standard. The low cost allows the technology to be widely deployed in wireless control and monitoring applications. Low power-usage allows longer life with smaller batteries. Mesh networking provides high reliability and more extensive range. The technology is intended to be simpler and less expensive than other WPAN's such as Bluetooth. ZigBee chip vendors typically sell integrated radios and microcontrollers with between $60 \mathrm{~KB}$ and $256 \mathrm{~KB}$ flash memory. ZigBee operates in the industrial, scientific and medical (ISM) radio bands; $868 \mathrm{MHz}$ in Europe, $915 \mathrm{MHz}$ in the USA and Australia, and $2.4 \mathrm{GHz}$ in most jurisdictions worldwide. Data transmission rates vary from 20 to 250 kilobits/second. The ZigBee network layer natively supports both star and tree typical networks, and generic mesh networks. Every network must have one coordinator device, tasked with its creation, the control of its 
parameters and basic maintenance. Within star networks, the coordinator must be the central node. Both trees and meshes allow the use of ZigBee routers to extend communication at the network.

\subsection{Micro Controller}

High-performance RISC CPU, Only 35 single word instructions to learn, Direct, indirect and relative addressing modes, Power-on Reset (POR), Power-up Timer (PWRT) and, Oscillator Start-up Timer (OST), Programmable code-protection, Low-power, high speed CMOS FLASH/EEPROM technology, In-Circuit Debugging via two pins, Single 5V In-Circuit Serial Programming capability, Wide operating voltage range: $2.0 \mathrm{~V}$ to $5.5 \mathrm{~V}$. High-performance RISC CPU: Only 35 single-word instruction to learn. Operating speed: DC$20 \mathrm{MHz}$ clock input, DC-200ns instruction cycle.[7]

\subsection{RS 232}

$\mathrm{PC}$ in general cannot directly communicate with peripherals that are available. The reason behind this is the difference in their working logic. PC generally works in positive logic. The microcontroller that actually acts as the peripheral here works in negative logic. It becomes important to change the logic between them when they communicate with each other. RS232 is very important for standard serial interfacing with PC where change of logic is achieved. PC communicates with peripherals through serial com 1 or com2.

\subsection{GSM Module}

PC: This unit contains the software components such as the server and security System through which the area security can be controlled and monitored. GSM Modem: It is a hardware component that allows the capability to send and receive SMS to and from the system. The communication with the system takes place via RS232 serial port. Cell phone can be attached at the place of GSM hardware but it limits the hardware functionality such as sending or receiving of SMS.[7]

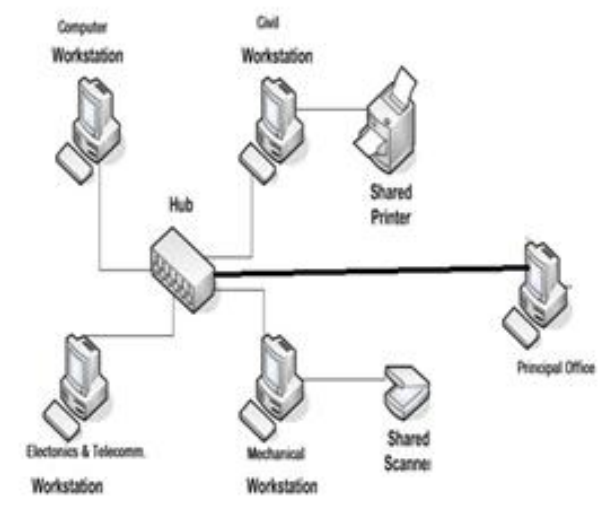

Fig. 3: LAN Networking for College

\subsection{LAN Networking}

A local-area network (LAN)is a computer network that spans a relatively small area. Most LANs are confined to a single building or group of buildings, however, one LAN can be connected to other LANs over any distance via telephone lines and radio waves. A system of LANs connected in this way is called a wide-area network (WAN). Most LANs connect workstations and personal computers. Each node (individual computer ) in a LAN has its own CPU with which it executes programs, but it also is able to access data and devices any where on the LAN. This means that many users can share expensive devices, such as laser printers, as well as data. Users can also use the LAN to communicate with each other, by sending e-mail or engaging in chat sessions. LANs are capable of transmitting data at very fast rates, much faster than data can be transmitted over a telephone line; but the distances are limited, and there is also a limit on the number of computers that can be attached to a single LAN.[9]

\subsection{Fingerprint Scanner}

A major factor in ensuring security is the unique identification of individuals, or the authentication that a person is who he or she claims to be. This must be done reliably, rapidly, non-intrusively and at reasonable cost. In the past, this has been done by methods such as security tokens (passports, badges, etc.), secure knowledge (passwords PIN codes, signature, etc.) or recognition by a guardian (doorkeeper). These traditional approaches are all limited with respect to the above criteria. A promising approach for the future is biometrics. Biometrics offers a convenient, reliable and low-cost means of identifying or authenticating individuals, and can 
be implemented in unsupervised and remote situations. Biometrics seeks to identify individuals uniquely by measuring certain physical and behavioral characteristics and extracting a sample (also called a sampled template or live template) from these measurements in a standard data format. This sample is compared with a template (also called an enrolled template or signature), based on the same characteristics, that has been established as the unique identity of that individual and stored in the security system. A close match between sample and template confirms the identity of the individual.[7]

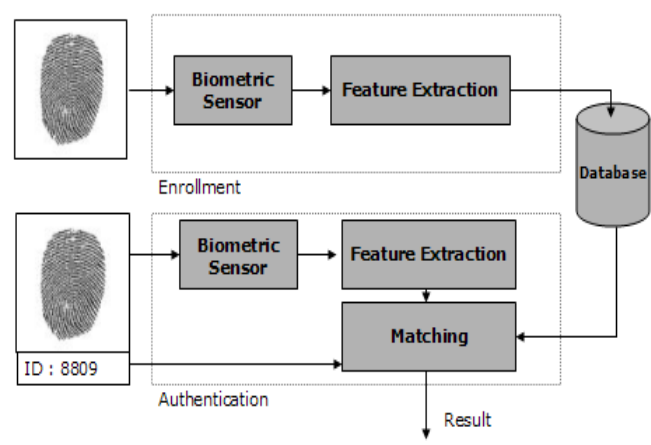

Fig 4: General biometric system

A fingerprint is the pattern of ridges and valleys on the surface of a fingertip. The End points and crossing points of ridges are called minutiae. It is a widely accepted assumption that the minutiae pattern of each finger is unique and does not change during one's life. Ridge endings are the points where the ridge curve terminates, and bifurcations are where a ridge splits from a single path to two paths at a Y-junction. Fingerprint processing includes two parts: fingerprint enrollment and fingerprint matching (the matching can be 1:1 or 1:N). When enrolling, user needs to enter the finger two times. The system will process the two time finger images, generate a template of the finger based on processing results and store the template.

When matching, user enters the finger through optical sensor and system will generate a template of the finger and compare it with templates of the finger library. For 1:1 matching, system will compare the live finger with specific template designated in the Module; for 1:N matching, or searching, system will search the whole finger library for the matching finger. In both circumstances, system will return the matching result, success or failure.

\subsection{Finger Chip Technology}

Finger Chip IC for fingerprint image capture combines detection and data conversion circuitry in a single rectangular CMOS die. It captures the image of a fingerprint as the finger is swept vertically over the sensor window. It requires no external heat, light or radio source. Most reliable biometric for uniquely identifying an individual. In spite of some recent legal challenges in the USA, they are still regarded as giving proof of identity beyond reasonable doubt in almost all cases. The majority of the biometric-based security systems in operation today are based on fingerprint recognition.[7]

\subsection{Serial Communication}

SCI is an abbreviation for Serial Communication Interface and, as a special subsystem, it exists on most microcontrollers. When it is not available, as is the case with PIC16F84, it can be created in software.

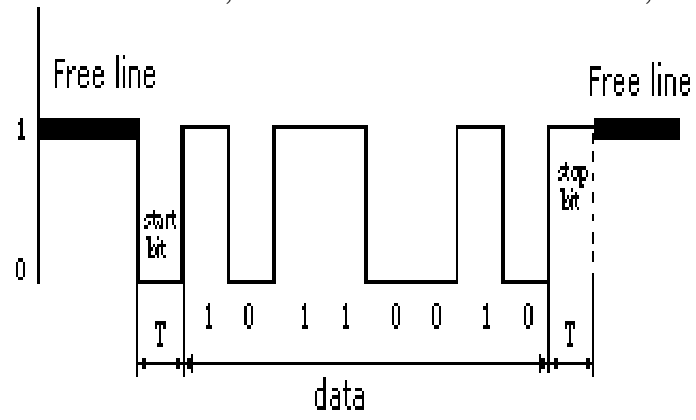

As with hardware communication, we use standard NRZ (Non Return to Zero) format also known as 8 (9)-N-1, or 8 or 9 data bits, without parity bit and with one stop bit. Free line is defined as the status of logic one. Start of transmission - Start Bit, has the status of logic zero. The data bits follow the start bit (the first bit is the low significant bit), and after the bits we place the Stop Bit of logic one. The duration of the stop bit 'T' 
depends on the speed of transmission and is adjusted according to the needs of the transmission. For the transmission speed of 9600 baud, $\mathrm{T}$ is $104 \mathrm{u}$

\section{Use Of Portable Devices}

\section{Results}

Teachers will verify his/her fingerprint on the device before giving it to students for marking attendance. After verifying the teacher's identity, software will ask for course and and other required information about the class which he or she is going to teach. Software will ask teacher the time after which device will not mark any attendance. This time can vary depending on the teacher's mood but our suggested value is 25 minutes. This is done to prevent late entrance of students. This step will hardly take few seconds. Then students will be given device for their fingerprint identification and attendance marking. In the continuation, teacher will start his/her lecture. Students will hand over the device to other students whose attendance is not marked. After 25 minutes or the time decided by teacher, device will not input any attendance. After the class is over, teacher will take device and will end the lecture. The main function of software running on the device will be fingerprint identification of students followed by report generation and sending reports to servers using $3 \mathrm{G}$ network. Other functions will be downloading and updating the database available on the device from central database repository.[8]

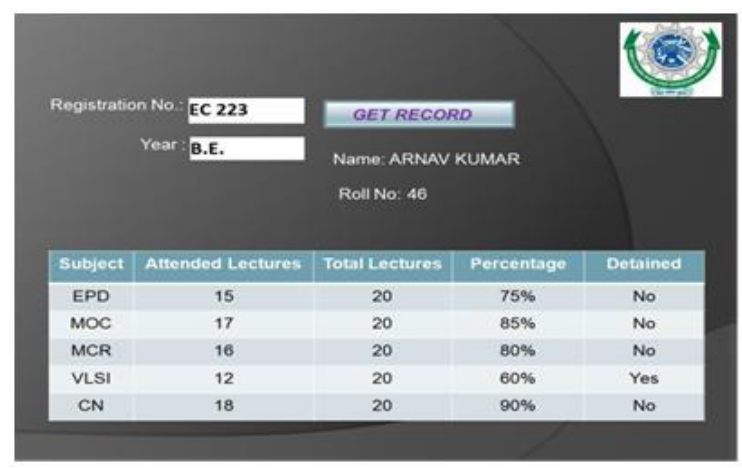

Fig. 4: Final Analysis of Project

The proposed report of each student will be obtained after the insertion of registration number and year. Above Figure represents one of the students attendance report after 1 month.

\section{Conclusion}

Thus the developed system provides fingerprint acquisition module and attendance management module in computer. It can realize automatically such functions as information acquisition of fingerprint, processing, wireless transmission, fingerprint matching, and attendance management .A fingerprint acquisition module and a wireless alarm module were designed by using the fingerprint sensor and GSM module respectively. The whole system was implemented wireless alarm through messages and internet in the GSM web. And the whole data which display on HOD cabin server that will be transmitted to the Principal office. This system is very advance towards Colleges. In order to achieve the simple and high real-time system, it realized low-cost and high-performance wireless fingerprint attendance function, which provided a new wireless fingerprint attendance system for enterprises and institutions. To design and develop a low cost and easily mountable advanced fingerprint attendance system using wireless technology for industries, colleges, hospitals, government offices etc..

\section{References}

[1]. Murizah Kassim, Hasbullah Mazlan, Norliza Zaini, Muhammad Khidhir Salleh "Web-based Student Attendance System using RFID Technology” 2012 IEEE.

[2]. B. Rasagna, Prof. C. Rajendra "SSCM: A Smart System for College Maintenance" International Journal of Advanced Research in Computer Engineering \& Technology, May 2012.

[3]. LI Jian-po, ZHU Xu-ning, LI Xue, ZHANG Zhi-ming "Wireless Fingerprint Attendance System Based on ZigBee Technology" 2010 IEEE.

[4]. O. Shoewu, O.A. Idowu "Development of Attendance Management System using Biometrics" The Pacific Journal of Science and Technology, May 2012.

[5]. "How does a fingerprint sensor work" Online Available: http://wiki.answers.com/Q/How_does_a_fingerprint_sens or_work [Accessed: Sept. 15, 2013].

[6]. "What is GSM?" Online Available:

[7]. http://www.tutorialspoint.com/gsm/gsm_overview.html [Accessed: Sept. 17, 2013].

[8]. L. Rajasekar, S. Vivek "Wireless Fingerprint Attendance System using ZigBee Technology" International Journal of Power Control Signal and Computation (IJPCSC), Vol3. No1. Jan-Mar 2012. 
[9]. Gunjan Talaviya, Rahul Ramteke, A.K.Shete, “ Wireless Fingerprint Based College Attendance System Using Zigbee Technology” International Journal of Engineering and Advanced Technology (IJEAT) ISSN: 2249 - 8958, Volume-2, Issue-3, February 2013

[10]. Rajan Patel1, Nimisha Patel2, Mona Gajjar3 "Online Students' Attendance Monitoring System in Classroom Using Radio Frequency Identification Technology: A Proposed System Framework” International Journal of Emerging Technology and Advanced Engineering Website: www.ijetae.com (ISSN 2250-2459, Volume 2, Issue 2, February 2012) 